\title{
Monitoring of the biological diversity of vegetation in the vicinity of Lake Kravtsovo
}

\author{
E.N. Seliverstova, and V.V. Volkova* \\ Federal state budgetary scientific institution «North-Caucasus Federal Agricultural Research Centre», \\ 356241, Mikhailovsk, Russia
}

\begin{abstract}
The purpose of this work was to conduct plant monitoring in the vicinity of Lake Kravtsovo within the framework of preserving animal and plant world biodiversity. Lake-swamp Kravtsovo - a unique object of the Stavropol upland, here you can study the diversity of almost half of the entire flora and fauna of Stavropol Territory. 139 species of vascular plants were registered in the studied territory, among which 11 are subject to protection Paeonia tenuifolia L., Cladium mariscus (L.) Ponl, Iris notha M. Bieb., I. halophila Pall., Adonis vernalis L., Calamagrostis canescens (Weber) Roth, Utricularia vulgaris L., Carex panicea L., C. ericetorum Pollich, Lythrum thesioides Bieb, Thelypteris palustris Schott. In the structure of the coenosis, the most part is occupied by forbs $-67 \%$, then cereals $-27 \%$, legumes in a smaller amount $-6 \%$. The composition and structure of the community are changed in some way by grazing of farm animals, land plowing and afforestation (a characteristic increase in the proportion of shrubs and trees). The initial stage of pasture degression and the associated increase in the role of associated grazing species (poisonous, prickly, poorly eaten and weedy) are observed. The horizontal structure of the vegetation cover is also heterogeneous.
\end{abstract}

\section{Introduction}

The preservation of animal and plant world biodiversity is a mandatory condition for sustainable development of the biosphere. This is a global problem. In 1992, the UN Conference on Environment and Development (UNCED) in Rio de Janeiro adopted the Convention on Biological Diversity in response to the acute problem of a significant reduction in the diversity of genetic resources. The extinction of species is associated both with natural factors (general climatic changes) and with a constant increase in anthropogenic pressure [1]. The most accessible control method is monitoring, the purpose of which is to record and analyze the results. Despite the originality of the flora of the Stavropol upland, its phytocenotic study remains incomplete. Data on the composition and structure of plant communities are insufficient and are not always accompanied by the publication of geobotanical descriptions [2, 3].

\footnotetext{
${ }^{*}$ Corresponding author: lotos026@mail.ru
} 
Vegetation surveys for individual steppe and forest formations are relevant for the purposes of regional typological work and the creation of a classification system for the vegetation cover of the Stavropol Territory. In-depth knowledge of the structure of vegetation cover and improvement of the typification of chorological units on the basis of geobotanical descriptions is one of the urgent tasks of botanical and geographical research. The results of our study are necessary both for mapping and zoning, and for assessing the actual phytocenotic diversity of taxa at different levels. In this connection, the purpose of this work is to monitor the biodiversity in the vicinity of the Lake Kravtsovo. This makes the work relevant for the inventory and monitoring of studies of steppe plant communities in the Stavropol Territory and allows to solve issues related to their protection.

\section{Materials and Methods}

Lake Kravtsovo is one of the oldest water bodies in the Stavropol Territory, formed during the ice age, it is a natural reserve of regional significance. The main object of interest of the lake is a one-of-a-kind floating island. In the northern part of its basin, there are underwater springs, there are pits at the bottom. Kravtsovo - lake-swamp is a unique object of the Stavropol upland and is of great value as an object of plant biodiversity studying, here the diversity of almost half of the entire flora and fauna of Stavropol can be studied. The total area is 169 hectares, including: land occupied by water - 73.91. It is located ten kilometers to the southwest of the city of Stavropol, on the upper terrace of the southern slope of the Grushevaya River, at an altitude of 560 meters above sea level. The basin has an elongated shape from east to west with a length of about $1.5 \mathrm{~km}$, a width of 500-600 m, a depth of up to $2.5 \mathrm{~m}$, in some places the coastline is steep up to $2.5 \mathrm{~m}$ high. The lake is fed by ground water, snow water, and precipitation. The flora of Lake Kravtsovo is characteristic of both open natural reservoirs and swamps, including peat bogs. In 2014, peat drifting "island", on which trees, shrubs, cattails, reeds, sedges, etc. grew, was washed up by strong wind gusts to one of the shores of the reservoir, where it broke up into four small fragments. Most of it joined the southwestern shore, while smaller parts were distributed along the western and southern shores. [4]

The main factual material was collected during the expedition research on the slopes and the shoreline of Lake Kravtsovo in 2013-2020. Cenotic surveys, collection and processing of field materials were carried out using generally accepted geobotanical methods $[5,6]$. The accounted sites had a size of $10 \times 10 \mathrm{~m}$ with a GPS-navigator marking. Algae, mosses, and lichens were not considered in the description. When studying the age conditions of populations, the following symbols were used: germs ( $\mathrm{pl})$, juvenile plants (j), immature (im), generative (g), and prussic (s). The vitality of the population was determined on a 5-point scale. The visual assessment of the abundance of the species in the cenosis is carried out in accordance with the Drude score scale [7].

The participation of the species in the composition of the phytocenosis was assessed by its cover. To indicate the total projective cover (TPC) of the herbage when adding phytocenoses, a uniform assessment scale was used [8].

The species names of plants in our work are given in accordance with the summary "Synopsis of the Flora of the Caucasus" and the Synopsis of the Flora of the Stavropol Territory [9]. The Latin names of the plants in our work are given in accordance with the summary of the S.K. Cherepanov [10].

Sozological studies of rare species were carried out by analyzing the categories of protection and the status of the species condition. The category of protection means the importance degree of preserving the gene pool of a given species and is divided into five categories: regional endemics, subendemics, relict species that have point ranges in the region and are rare beyond its borders: glacial and xerothermal relicts, species that do not belong to the first four categories - rare for natural reasons [11]. 
The places of species growth were determined by spot mapping method. Monitoring of rare species in culture and nature was carried out according to the "Program and methodology of observations of plant species cenopopulations of the Red Book of the USSR " [12].

\section{Results and Discussion}

The Stavropol Territory, where the Lake Kravtsovo is located, is located in the center of the North Caucasus. It is characterized by a constant change of landscapes and different natural and climatic conditions. According to the conditions of moisture supply, the Stavropol Territory is located in the $\mathrm{V}$ moderately humid region, according to heat supply - an insufficiently hot subdistrict with HTC of 1.1-1.3. This is a zone of unstable moisture supply - from 650 to 700 $\mathrm{mm}$ of precipitation falls per year. During the growing season, the relative humidity ranges from 54-56 \% [13]. During the years of research, acute uneven precipitation, mainly of a stormwater nature, and high temperatures in the summer were observed.

2019 and 2020 were warmer than the long-term norm, characterized by atmospheric and soil drought. The average daily air temperature for the year is $9.9^{\circ} \mathrm{C}$, long-term $-+7.5{ }^{\circ} \mathrm{C}$. The maximum reached plus $35.0{ }^{\circ} \mathrm{C}$, the minimum - minus $20.0{ }^{\circ} \mathrm{C}$. Precipitation did not fall regularly, and had a stormy character. $518.0 \mathrm{~mm}$ of precipitation fell, which is less than the long-term norm $(720.0 \mathrm{~mm})$. Of course, this also affected the herbage. According to the literature data, $15 \%$ of the territory of the southern coast is covered with broad-leaved forest (Fagus orientalis Lipsky, Salix caprea L., Quercus robur L.), and 10\% of the eastern slope is covered with shrubs (Crataegus monogyna Jacq, Crataegus pentagyna Waldst. \& Kit. ex Willd., Prunus spinosa L., Sambucus nigra f. laciniata (L.) Zabel, Rosa sp.).

In the southern and northern parts of the water protection zone, the natural grassland of the meadow steppe has been preserved and grows, in which Paeonia tenuifolia L., Cladium mariscus (L.) Ponl listed in the Red Book of the Russian Federation [11], and Iris notha M. are found. Bieb., Iris halophila Pall., Adonis vernalis L., Calamagrostis canescens (Weber) Roth, Utricularia vulgaris L., Carex panicea L., Carex ericetorum Pollich, Lythrum thesioides Bieb, Thelypteris palustris Schott, listed in the Red Book of the Stavropol Territory, as well as Poa pratensis L., Geranium sanguineum L., Fragaria viridis (Duchesne) Weston, Medicago falcata L., Euphorbia iberica Boiss., Polygala caucasica Rupr., Typha angustifolia L., Phragmites communis (Cav.) Trin. ex Steud. with dense projective coverage from 81 to $98 \%$.

The western slope of the lake is an elongated narrow area from south to west with a total area of 1.5 ha with xerophilic vegetation (the projective cover is not dense up to $40 \%$ ) with a predominance of Carex humilis Leys., C. supina Willd. ex Wahlenb., Phlum phleoides (L.) H. Karst., Festuca ovina L., Bromopsis inermis (Leyss.) Holub, etc.

The composition and structure of the community are changed in some way by grazing of farm animals, land plowing and afforestation (a characteristic increase in the proportion of shrubs and trees). The initial stage of pasture degression and the associated increase in the role of associated grazing species (poisonous, prickly, poorly eaten and weedy) are observed. The horizontal structure of the vegetation cover is heterogeneous.

The first tier, up to $2 \mathrm{~m}$ high, consists of Typha angustifolia, Phragmites communis, Conium maculatum, Verbascum lychnitis etc. The second tier, up to 60-70 cm high, consists of Medicago falcata, Iris notha, Iris halophile, Glycyrrhiza glabra, Lomelosia micrantha, Centaurea orientalis. The third tier $-30-40 \mathrm{~cm}$ high, consists of Paeonia tenuifolia, Artemisia absinthium, Consolida paniculata, Salvia aethiopis, Phlomis pungens, Eryngium campestre; Poterium polygamum, Achillea nobilis, Echium vulgare, etc., the fourth tier is up to $20 \mathrm{~cm}$ high - Artemisia austriaca, Festuca valesiaca, the fifth tier - we did not take into account mosses, lichens and algae. In the cenosis structure, a large part is occupied by forbs, including woody and weedy vegetation - $67 \%$, then cereals - at least $27 \%$, legume components in a smaller amount $-6 \%$. 


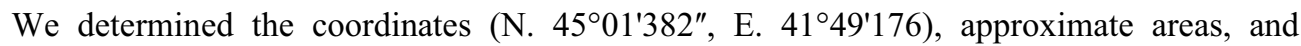
described the populations of sedges and peonies and other rare species. The results of the surveys are presented in table 1. During the period of 2013-2014, the number and age composition did not change much. Fires in 2014 led to a decrease in the number of individuals of Carex lasiocarpa, C. diluta, C. acuta, Iris halophila, I. notha, Paeonia tenuifolia.

Table 1. Species composition and characteristics of steppe phytocenosis

\begin{tabular}{|c|c|c|c|c|}
\hline \multirow[t]{2}{*}{ No. } & \multirow{2}{*}{$\begin{array}{l}\text { Species, floristic group } \\
\text { Species, floristic group }\end{array}$} & \multicolumn{2}{|c|}{$\begin{array}{l}\text { Abundance acc. to Drude } \\
\text { Abundance of Drude }\end{array}$} & \multirow{2}{*}{$\begin{array}{c}\text { Life form } \\
\text { (biomorph) } \\
\text { Life form } \\
\text { (biomorph) }\end{array}$} \\
\hline & & 2014-2016 & 2020 & \\
\hline \multicolumn{5}{|c|}{ Cereals and Carex/ Poa and Carex } \\
\hline 1. & Carex humilis Leys & $\mathrm{sp}^{1}$ & $\mathrm{sp}^{1}$ & $\mathrm{~Pa}$ \\
\hline 2. & C. michelii Host & $\mathrm{sp}^{1}$ & $\mathrm{sp}^{1}$ & $\mathrm{~Pa}$ \\
\hline 3. & C. tomentosa $\mathrm{L}$. & $\mathrm{sp}^{2}$ & $\mathrm{sp}^{1}$ & $\mathrm{~Pa}, \mathrm{St}$ \\
\hline 4. & C. pseudocyperus $\mathrm{L}$ & [sol] & - & $\mathrm{Ab}$ \\
\hline 5. & C. diluta Bieb. & un & - & $\mathrm{Ab}$ \\
\hline 6. & C. melanostachya Bieb. & {$\left[\mathrm{sp}^{2}\right]$} & {$\left[\mathrm{sp}^{2]}\right.$} & $\mathrm{Aa}$ \\
\hline 7. & C. riparia Curt & $\mathrm{sp}^{1}$ & $\mathrm{sp}^{2}$ & $\mathrm{Ab}$ \\
\hline 8. & C. panicea $\mathrm{L}$. & [sol] & - & $\mathrm{Aa}$ \\
\hline 9. & C. vulpina $\mathrm{L}$. & un & - & $\mathrm{Ab}$ \\
\hline 10. & C. hirta L. & {$\left[\mathrm{sp}^{1}\right]$} & [sol] & $\mathrm{Aa}$ \\
\hline 11. & C. praecox Schreb. & $\mathrm{sp}^{1}$ & sol & St \\
\hline 12. & C. otrubae Podp. & sol & sol & $\mathrm{Ab}$ \\
\hline 13. & C. acuta $\mathrm{L}$. & [sol] & - & $\mathrm{Ab}$ \\
\hline 14. & C. lasiocarpa Ehrh. & {$[\mathrm{sol}]$} & - & $\mathrm{Ab}$ \\
\hline 15. & C. vesicaria $\mathrm{L}$. & [sol] & - & $\mathrm{Ab}$ \\
\hline 14. & C. acutiformis Ehrh. & $\mathrm{sp}^{2}$ & $\mathrm{sp}^{1}$ & $\mathrm{Ab}$ \\
\hline 15. & Alopecurus arundinaceus Poir. & $\mathrm{sp}^{1}$ & $\mathrm{sp}^{1}$ & $\mathrm{Aa}$ \\
\hline 16. & Bothriochloa ischaemum (L.) Keng & $\mathrm{sp}^{1}$ & $\mathrm{sp}^{3}$ & St \\
\hline 17. & Brachypodium pinnatum (L.) Beauv. & $\mathrm{sp}^{1}$ & sol & $\mathrm{S}$ \\
\hline 18. & Bromopsis inermis (Leyss.) Holub. & $\mathrm{sp}^{1}$ & sol & St \\
\hline 19. & B. riparia (Rehmann) Holub. & $\mathrm{sp}^{1}$ & $\mathrm{sp}^{1}$ & $\mathrm{St}$ \\
\hline 20. & $\begin{array}{l}\text { Calamagrostis canescens (Weber) } \\
\text { Roth }\end{array}$ & $\mathrm{sp}^{1}$ & $\mathrm{sp}^{1}$ & $\mathrm{Aa}$ \\
\hline 21. & $\begin{array}{l}\text { C. pseudophragmites (Haller f.) } \\
\text { Koeler }\end{array}$ & [sol] & [sol] & Aa \\
\hline 22. & Catabrosa aguatica (L.) P. Beauv. & $\mathrm{sp}^{1}$ & $\mathrm{sp}^{2}$ & $\mathrm{Aa}$ \\
\hline 23. & Digitaria sanguinalis (L.) Scop. & $\mathrm{Sp}^{1}$ & $\mathrm{sp}^{1}$ & $\mathrm{~Pa}, \mathrm{Rb}$ \\
\hline 24. & Echinochloa crus-galli (L.) P. Beauv. & $\mathrm{sp}^{1}$ & $\mathrm{sp}^{2}$ & $\mathrm{~Pa}, \mathrm{Rb}$ \\
\hline 25. & Elytrigia repens (L.) Nevski & $\mathrm{sp}^{1}$ & $\mathrm{sp}^{3}$ & $\mathrm{~Pa}, \mathrm{St}$ \\
\hline 26. & Festuca valesiaca Gaudin & $\mathrm{sp}^{1}$ & $\mathrm{sp}^{3}$ & $\mathrm{St}$ \\
\hline 27. & Glyceria maxima (Hartm.) Holmb. & $\mathrm{sp}^{1}$ & sol & $\mathrm{Aa}$ \\
\hline 28. & Koeleria macrantha (Ledeb.) Schult. & $\mathrm{sp}^{1}$ & $\mathrm{sp}^{2}$ & St \\
\hline 29. & Melica transsilvanica Schur & $\mathrm{sp}^{1}$ & sol & St \\
\hline 30. & Phleum pratense L. & $\mathrm{sp}^{1}$ & sol & $\mathrm{Pa}$ \\
\hline 31. & Poa palustris L. & $\mathrm{sp}^{2}$ & $\mathrm{sp}^{2}$ & $\mathrm{Aa}$ \\
\hline 32. & Setaria verticillata (L.) P. Beauv. & $\mathrm{sp}^{1}$ & $\mathrm{sp}^{1}$ & $\mathrm{~Pa}, \mathrm{Rb}$ \\
\hline 33. & S. viridis (L.) P. Beauv. & $\mathrm{sp}^{1}$ & $\mathrm{sp}^{1}$ & $\mathrm{~Pa}, \mathrm{Rb}$ \\
\hline 34. & Stipa lessingiana Trin. \& Rupr. & $\mathrm{sp}^{1}$ & $\mathrm{sp}^{1}$ & $\mathrm{St}$ \\
\hline 35. & S. pulcherrima K. Koch & $\mathrm{sp}^{1}$ & $\mathrm{sp}^{1}$ & $\mathrm{St}$ \\
\hline 36. & S. ucrainica P.A. Smirn. & $\mathrm{sp}^{1}$ & $\mathrm{sp}^{1}$ & $\mathrm{St}$ \\
\hline 37. & Tragus racemosus (L.) All. & $\mathrm{sp}^{1}$ & $\mathrm{sp}^{1}$ & $\mathrm{Db}, \mathrm{Ra}$ \\
\hline
\end{tabular}


Legumes / Pulsus

\begin{tabular}{|c|l|r|}
\hline 38. & Astragalus bungeanus Boiss. & [sol \\
\hline 39. & A. pseudotataricus Boriss. & sol \\
\hline 40. & $\begin{array}{l}\text { Chamaecytisus ruthenicus (Fisch. ex. } \\
\text { Woloszcz.) Klaskova }\end{array}$ & {$[\mathrm{sp}$} \\
\hline 41. & Glycyrrhiza glabra L. & $\mathrm{sp}$ \\
\hline 42. & Hedysarum biebersteinii Zertova & $\mathrm{so}$ \\
\hline 43. & Medicago falcata L. & $\mathrm{sp}$ \\
\hline 44. & Melilotus officinalis (L.) Pall. & {$\left[\mathrm{sp}^{1}\right.$} \\
\hline 45. & Trifolium repens L. & $\mathrm{sp}$ \\
\hline 46. & Vicia sepium L. & $\mathrm{sp}$ \\
\hline
\end{tabular}

Forbs / Raznotravie

\begin{tabular}{c|c|c|}
\hline$[\mathrm{sol}]$ & \multicolumn{2}{|c|}{} \\
\hline sol & {$[\mathrm{sol}]$} & $\mathrm{St}$ \\
\hline$\left[\mathrm{sp}^{1}\right]$ & {$\left[\mathrm{sp}^{2}\right]$} & $\mathrm{St}, \mathrm{Db}$ \\
\hline $\mathrm{sp}^{1}$ & $\mathrm{sp}^{1}$ & $\mathrm{St}$ \\
\hline $\mathrm{sol}$ & - & $\mathrm{St}$ \\
\hline $\mathrm{sp}^{1}$ & $\mathrm{sp}^{2}$ & $\mathrm{Da}$ \\
\hline $\left.\mathrm{sp}^{1}\right]$ & $\mathrm{sp}^{2}$ & $\mathrm{Rb}$ \\
\hline $\mathrm{sp}^{1}$ & $\mathrm{sp}^{2}$ & $\mathrm{~Pa}, \mathrm{Rb}$ \\
$\mathrm{sp}^{1}$ & $\mathrm{sp}^{2}$ & $\mathrm{~Pa}, \mathrm{Rb}$ \\
\hline
\end{tabular}

\begin{tabular}{|l|l|l}
\multicolumn{3}{|c}{ Forbs / Raznotravie } \\
\hline 47. & Juglans regia L. & \\
\hline 48. & Malus domestica Borkh. & \\
\hline 49. & Populus alba L. &
\end{tabular}

49. Populus alba L.

50. $\quad$ Armeniaca vulgaris Lam.

51. Crataegus monogyna Jacq

52.

C. pentagyna Waldst. \& Kit. ex

52. Willd.

53. $\quad$ Elaeagnus orientalis L.

54. $\quad$ Prunus spinosa L.

55. $\quad$ Pyrus caucasica Fed.

56. Rosa sp.

$57 . \quad$ Rosa spinosissima L.

58. Rubus caesius L.

$59 . \quad$ Salix caprea L.

60. Sambucus nigra f. laciniata (L.)

Zabel

61. Thelypteris palustris Schott

$62 . \quad$ Aster amellus L.

$63 . \quad$ Adonis vernalis $\mathrm{L}$.

64. Agrimonia eupatoria L.

65. Ajuga genevensis L.

66. Alcea rugosa Alef.

67. Alisma lanceolatum With.

$68 . \quad$ A. plantago-aquatica L.

69. Ambrosia artemisiifolia L.

$70 . \quad$ Artemisia absinthium L.

$71 . \quad$ A. vulgaris L.

72. Atriplex littoralis L.

73. Bolboschoenus maritimus (L.) Palla

74. Butomus umbellatus L.

$75 . \quad$ Campanula praealta Galushko

$76 . \quad$ Carduus crispus L.

$77 . \quad$ C. nutans L.

$78 . \quad$ C. thoermeri Weinm.

$79 . \quad$ Centaurea orientalis L.

80 . Conium maculatum L.

81. Chelidonium majus L.

82. Cichorium intybus L.

83. Cladium mariscus (L.) Pohl

84. Crocus reticulatus Stev. ex Adams

85. Dictamnus caucasicus (Fisch. \& C.A. Mey.) Grossh.

\begin{tabular}{l|l}
- & $\mathrm{u}$ \\
\hline- &
\end{tabular}

un $\quad \mathrm{Pa}$

sol]

[sol]

$\mathrm{Pa}$

$-$

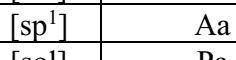

sol

[sol]

sol]
$\mathrm{sp}^{1}$

sol

$\mathrm{sp}^{1}$

$\mathrm{Pa}, \mathrm{St}$

sol

$\mathrm{sp}^{1}$

sp

sol

sol

sol

$-$

$\left.\mathrm{sp}^{1}\right]$

-

\begin{tabular}{|l|l}
$\mathrm{sp}$ \\
$\mathrm{Sp}$
\end{tabular}

$\mathrm{sp}^{1} \quad \mathrm{Db}$

\begin{tabular}{l|c}
$\mathrm{Sp}^{3}$ & $\mathrm{~Pa}, \mathrm{~S}$ \\
\hline $\mathrm{Sol}]$ & $\mathrm{S}$ \\
\hline
\end{tabular}

$86 . \quad$ Daucus carota L.

\begin{tabular}{|c|c|c|}
\hline - & [sol] & $\mathrm{S}, \mathrm{Rb}$ \\
\hline$\left[\mathrm{sp}^{1}\right]$ & {$\left[\mathrm{Sp}^{3}\right]$} & $\mathrm{Ab}$ \\
\hline sol & $\mathrm{Sp}^{1}$ & $\mathrm{~Pa}, \mathrm{St}$ \\
\hline$\left[\mathrm{sp}^{1}\right]$ & {$\left[\mathrm{sp}^{1}\right]$} & $\mathrm{Pa}, \mathrm{St}$ \\
\hline $\mathrm{sp}^{1}$ & $\mathrm{Sp}^{2}$ & $\mathrm{~Pa}, \mathrm{St}$ \\
\hline sol & $\mathrm{sp}^{1}$ & $\mathrm{~Pa}$ \\
\hline sol & sol & $\mathrm{Pa}, \mathrm{St}$ \\
\hline [sol] & [sol] & $\mathrm{Aa}$ \\
\hline$\left[\mathrm{sp}^{1}\right]$ & {$\left[\mathrm{sp}^{1}\right]$} & $\mathrm{Aa}$ \\
\hline- & $\mathrm{sp}^{1}$ & $\mathrm{Rb}, \mathrm{Pa}$ \\
\hline sol & $\mathrm{sp}^{1}$ & $\mathrm{Rb}$ \\
\hline sol & $\mathrm{sp}^{1}$ & $\mathrm{Rb}$ \\
\hline sol & $\mathrm{sp}^{1}$ & $\mathrm{Dc}$ \\
\hline Cop $^{1}$ & $\mathrm{sp}^{1}$ & $\mathrm{Aa}, \mathrm{Ab}$ \\
\hline [sol] & - & $\mathrm{Ab}$ \\
\hline sol & sol & $\mathrm{Pa}$ \\
\hline $\mathrm{sp}^{1}$ & $\mathrm{sp}^{1}$ & $\mathrm{Rb}$ \\
\hline sol & $\mathrm{sp}^{1}$ & $\mathrm{Rb}$ \\
\hline [sol] & $\mathrm{sp}^{1}$ & $\mathrm{Rb}$ \\
\hline $\mathrm{sp}^{1}$ & $\mathrm{sp}^{1}$ & $\mathrm{St}$ \\
\hline [sol] & [sol] & $\mathrm{Rb}$ \\
\hline- & [sol] & $\mathrm{Rb}, \mathrm{S}$ \\
\hline $\mathrm{sp}^{1}$ & $\mathrm{sp}^{1}$ & $\mathrm{Rb}, \mathrm{Pa}$ \\
\hline [Sol] & [sol] & $\mathrm{Ab}$ \\
\hline sol & sol & $\mathrm{Pa}, \mathrm{St}$ \\
\hline $\mathrm{Sp}^{2}$ & {$\left[\mathrm{sp}^{1}\right]$} & $\mathrm{S}, \mathrm{Pb}$ \\
\hline $\mathrm{sp}^{1}$ & $\mathrm{Sp}^{2}$ & $\mathrm{Rb}, \mathrm{Pa}$ \\
\hline
\end{tabular}




\begin{tabular}{|c|c|c|c|c|}
\hline 87. & Delphinium consolida L. & sol & sol & $\mathrm{St}$ \\
\hline 88. & Echium russicum J.F. Gmel. & sol & sol & $\mathrm{Pa}$ \\
\hline 89. & $\begin{array}{l}\text { Eleocharis palustris (L.) Roem. \& } \\
\text { Schult. }\end{array}$ & [sol] & [sol] & $\mathrm{Aa}$ \\
\hline 90. & Epilobium hirsutum L. & $\mathrm{sp}^{1}$ & $\mathrm{sp}^{1}$ & $\mathrm{Aa}$ \\
\hline 91. & Euphorbia iberica Boiss. & sol & sol & $\mathrm{Rb}, \mathrm{St}$ \\
\hline 92. & E. stepposa Zoz ex Prokh. & sol & sol & St \\
\hline 93. & Falcaria vulgaris Bernh. & $\mathrm{sp}^{1}$ & $\mathrm{Sp}^{2}$ & $\mathrm{~Pa}, \mathrm{St}$ \\
\hline 94. & Filipendula vulgaris Moench & $\mathrm{sp}^{1}$ & $\mathrm{sp}^{1}$ & $\mathrm{~Pa}, \mathrm{St}$ \\
\hline 95. & Fragaria viridis (Duchesne) Weston & $\mathrm{sp}^{1}$ & sol & $\mathrm{Pa}$ \\
\hline 96. & Galium aparine $\mathrm{L}$. & sol & $\mathrm{sp}^{1}$ & $\mathrm{Ra}, \mathrm{Rb}$ \\
\hline 97. & Geranium sanguineum L. & sol & $\mathrm{sp}^{1}$ & $\mathrm{~Pa}$ \\
\hline 98. & Hypericum elegans Steph. ex Willd. & $\mathrm{Sp}^{2}$ & $\mathrm{Sp}^{2}$ & St \\
\hline 99. & Inula helenium L. & [sol] & {$\left[\mathrm{sp}^{1}\right]$} & $\mathrm{Pa}, \mathrm{Aa}$ \\
\hline 100. & Iris notha Bieb. & sol & sol & $\mathrm{Pa}$ \\
\hline 101. & I. halophila Pall. & sol & sol & $\mathrm{D}, \mathrm{St}$ \\
\hline 102. & Juncus effusus L. & [sol] & $\mathrm{sp}^{1}$ & $\mathrm{Aa}$ \\
\hline 103. & J. inflexus L. & [sol] & {$\left[\mathrm{sp}^{1}\right]$} & Aa \\
\hline 104. & J. gerardii Loisel. & [sol] & [sol] & $\mathrm{Aa}$ \\
\hline 105. & Leonurus quinquelobatus Gilib. & sol & sol & $\mathrm{Rb}$ \\
\hline 106. & Lythrum salicaria L. & sol & $\mathrm{sp}^{1}$ & $\mathrm{Aa}$ \\
\hline 107. & Leontodon hispidus L. & sol & sol & $\mathrm{Rb}, \mathrm{Pa}$ \\
\hline 108. & $\begin{array}{l}\text { Lomelosia micrantha (Desf.) Greuter } \\
\text { \& Burdet }\end{array}$ & sol & $\mathrm{sp}^{1}$ & St \\
\hline 109. & Muscari muscarimi Medikus & {$\left[\mathrm{sp}^{1}\right]$} & [sol] & $\mathrm{Pa}, \mathrm{St}$ \\
\hline 110. & Oberna behen (L.) Ikonn. & $\mathrm{sp}^{1}$ & $\mathrm{sp}^{1}$ & $\mathrm{Rb}, \mathrm{S}$ \\
\hline 111. & Origanum vulgare L. & [sol] & [sol] & $\mathrm{Pa}, \mathrm{St}$ \\
\hline 112. & Ornithogalum ponticum Zahar. & $\mathrm{sp}^{1}$ & sol & $\mathrm{Pa}$ \\
\hline 113. & Paeonia tenuifolia $\mathrm{L}$. & [sol] & {$\left[\mathrm{sp}^{1}\right]$} & $\mathrm{Pa}, \mathrm{St}$ \\
\hline 114. & $\begin{array}{l}\text { Petasites hybridus (L.) Gaertn. B. } \\
\text { Mey. \& Scherb. }\end{array}$ & {$[\mathrm{sol}]$} & [sol] & $\mathrm{S}, \mathrm{Aa}$ \\
\hline 115. & Phlomis pungens Willd. & sol & sol & St \\
\hline 116. & $\begin{array}{l}\text { Phragmites communis (Cav.) Trin. ex } \\
\text { Steud. }\end{array}$ & $\operatorname{cop}^{1}$ & $\operatorname{cop}^{3}$ & $\mathrm{Aa}, \mathrm{Ab}$ \\
\hline 117. & Plantago maxima Juss. ex Jacq. & sol & $\mathrm{sp}^{1}$ & $\mathrm{Ra}, \mathrm{Rb}$ \\
\hline 118. & Plantago media L. & sol & sol & $\mathrm{Ra}$ \\
\hline 119. & Polygala caucasica Rupr. & $\mathrm{sp}^{1}$ & $\mathrm{sp}^{1}$ & $\mathrm{~Pa}$ \\
\hline 120. & Primula macrocalyx Bunge & $\mathrm{sp}^{1}$ & $\mathrm{sp}^{1}$ & $\mathrm{~S}, \mathrm{~Pa}$ \\
\hline 121. & $\begin{array}{l}\text { Psephellus dealbatus (Willd.) K. } \\
\text { Koch }\end{array}$ & {$[\mathrm{sol}]$} & [sol] & $\mathrm{Pa}$ \\
\hline 122. & Rumex acetosa L. & sol & sol & $\mathrm{Pb}$ \\
\hline 123. & Salvia verticillata $\mathrm{L}$. & $\mathrm{sp}^{1}$ & sol & $\mathrm{Rb}, \mathrm{Pa}$ \\
\hline 124. & Schoenoplectus lacustris (L.) Palla & $\mathrm{sp}^{1}$ & $\mathrm{sp}^{3}$ & $\mathrm{Aa}, \mathrm{Ab}$ \\
\hline 125. & Sparganium erectum L. & $\mathrm{sp}^{1}$ & $\mathrm{sp}^{1}$ & $\mathrm{Ab}$ \\
\hline 126. & Sonchus arvensis L. & $\mathrm{sp}^{1}$ & $\mathrm{sp}^{2}$ & $\mathrm{Rb}, \mathrm{Ra}$ \\
\hline 127. & Stellaria neglecta Weihe & sol & sol & $\mathrm{Pb}$ \\
\hline 128. & Tanacetum vulgare $\mathrm{L}$. & sol & sol & $\mathrm{Rb}, \mathrm{Pa}$ \\
\hline 129. & Taraxacum officinale F.H. Wigg. & sol & sol & $\mathrm{Rb}, \mathrm{Pa}$ \\
\hline 130. & Thalictrum minus L. & $\mathrm{sp}^{1}$ & $\mathrm{sp}^{2}$ & $\mathrm{~Pa}, \mathrm{St}$ \\
\hline 131. & Thymus marschallianus Willd. & {$\left[\mathrm{sp}^{1}\right]$} & {$\left[\mathrm{sp}^{1}\right]$} & $\mathrm{Pa}, \mathrm{St}$ \\
\hline 132. & Typha angustifolia $\mathrm{L}$. & $\mathrm{sp}^{1}$ & $\mathrm{sp}^{2}$ & $\mathrm{Ab}$ \\
\hline 133. & T. latifolia $\mathrm{L}$. & $\mathrm{sp}^{1}$ & $\mathrm{sp}^{2}$ & $\mathrm{Ab}$ \\
\hline 134. & Urtica dioica $\mathrm{L}$. & - & $\mathrm{sp}^{1}$ & $\mathrm{Rb}$ \\
\hline 135. & Verbascum thapsus $\mathrm{L}$. & sol & sol & $\mathrm{Rb}$ \\
\hline
\end{tabular}




\begin{tabular}{|l|l|c|c|c|}
136. & V. lychnitis L. & sol & sol & $\mathrm{Pa}$ \\
\hline 137. & Veronica anagallis-aquatica L. & sol & sol & $\mathrm{Aa}, \mathrm{Ab}$ \\
\hline 138. & V. spicata L. & sol & $\mathrm{sp}^{1}$ & $\mathrm{ST}$ \\
\hline 139. & Vinca herbacea Waldst. \& Kit. & - & {$\left[\mathrm{sp}^{1}\right]$} & $\mathrm{Pa}$ \\
\hline
\end{tabular}

Symbols: $\mathrm{S}$ - forest, $\mathrm{Rb}$ - ruderal, $\mathrm{Ra}$-segetal, $\mathrm{Pa}$ - plain, $\mathrm{St}$ - steppe, $\mathrm{Ab}$ - hydrophilic, $\mathrm{Aa}-$ hygrophilic, $\mathrm{Db}$ - psammophilic, $\mathrm{Da}$ - calcepetrophilic; [] - local location, Cop ${ }^{3}$ - very abundant; $\mathrm{Cop}^{2}-$ abundant; $\mathrm{Cop}^{1}$ - quite abundant; $\mathrm{Sp}^{3}$ - abundantly scattered, $\mathrm{Sp}^{2}$ - scattered, $\mathrm{Sp}^{1}$ - little scattered; Sol single (very little); Un - in a single unit on the site.

According to the literature and herbarium data, 20 species of sedge (Carex hallerana Asso, C. vesicaria L., C. ericetorum Poll., C. disticha Huds, etc.) grew in the coastal zone of the lake $[14,15]$. During the entire period of our expeditionary activity, we did not find C. ericetorum Poll., C. disticha Huds. During the survey of the surrounding area and the coastal zone of the lake in 2014-2016, 16 species of the genus Carex were identified, and in 2020 - only 8. Until 2019, the sedge populations are stable, vital - 5 points, the distribution of the group is uneven. They grow together with Astragalus bungeanus (Sol), Hedysarum biebersteinii (Sol), Iris halophila (Sp), Astragalus pseudotataricus (Sol), Stipa pulcherrima (Sp) and other species in the associations Carex humilis- vario-herbosum, Carex praecox - vario-herbosum, Carex caryophyllea - vario-herbosum, Carex tomentosa-vario-herbosum, , where forbs predominate. In 2020 , we observed a decrease in the number of generative individuals by $50 \%$ compared to previous years (fires, lack of moisture in the dry 2019-2020 years).

Rare species Paeonia tenuifolia, Adonis vernalis, and Iris halophila, I. notha grow on the western slope in the forb-fescue-feather grass steppe on fertile, thick medium-humus, leached and saline clay chernozems. In 2014, due to the fire, the number of germs and vegetative individuals decreased by $20 \%$, but by 2020 , the population recovered, thanks to good seed productivity. Environmental factors that affect the vegetation cover are economic activity (land development), decrease in precipitation and wind erosion, which leads to decrease in the height of the fertile soil layer [3]. These factors cause an accelerated change in plant associations in terms of diversity of composition and structure, and the appearance of a large number of weeds and woody vegetation, which happened in 2020 . The number of weeds and woody vegetation increased due to anthropogenic factors (fires and increase in temperature with decrease in precipitation): Elytrigia repens, Atriplex littoralis, Urtica dioica, Ambrosia artemisiifolia, etc., trees - Pyrus caucasica, Malus domestica, shrubs - Crataegus monogyna, C. Pentagyna, Prunus spinosa, species Rosa, etc.

\section{Conclusions}

1. 139 species of vascular plants were registered in the studied territory, among which 9 are subject to regional protection (subendemics, xerothermal relics, vulnerable and heavily exploited species).

2. The results obtained allow to assess the real phytocenotic diversity of the surroundings of the Lake Kravtsovo, as well as to update further work on the inventory and monographic generalization of vegetation materials of the Stavropol Territory. As well as monitoring conducted in relation to rare species, it will allow to conduct environmental activities in relation to them.

3. The phyto-diversity of vegetation in the vicinity of the Lake Kravtsovo confirms the high environmental significance of plant communities for the region. 


\section{References}

1. V.N. Belous, V.V. Volkova, On the issue of phyto-diversity of the water-swamp complex of the Stavropol upland (on the example of the lake-swamp Kravtsovo), Actual problems of chemistry, biology and biotechnology materials of the X All-Russian Scientific Conference, 19-22 (2016) http://elibrary.ru/item.asp?id=26258188

2. Global Plant Conservation Strategy, Botanic Gardens Conservation International (BGCI) (M, 16, 2004)

3. E.N. Seliverstova, V.V. Khrapach, V.V. Volkova, N.V. Shegrinets, The Results of Plant Monitoring in the Semistozhki Area of Stavropol Region, Interhational scientific and practical conference "AgroSMART-Smart solutions for agriculture», KnE Life Sciences, 1013-1022 (2019), DOI 10.188502/ kls. V4i14.5700

4. L. Ivanov, Red Book of the Stavropol Territory: Rare and endangered species of plants and animals (Stavropol, Polygraphservice, 384, 2002)

5. A.A. Korchagin, Species (floral) composition of plant communities and methods of its study, Field geobotany (M., L.: Nauka, 3, 39-58, 1964)

6. A.A. Yunatov, Types and content of geobotanical research. Selection of test areas and laying of environmental profiles, Field geobotanics, (M.; L.: Nauka, 3, 9-36, 1964)

7. V.M. Poniatovskaya, Accounting for abundance and features of species placement in natural plant communities, Field geobotanics, (M.; L.: Nauka, 3, 209-299, 1964)

8. B.M. Mirkin, G.S. Rosenberg, Explanatory dictionary of modern phytocenology (M.: Nauka, 134 (1983)

9. A.L. Ivanov, Synopsis of the flora of the Stavropol Territory, Ministry of Education RF (Stavropol, Pub. Stavropol State University, 199, 2001)

10. S.K. Cherepanov, Vascular plants of Russia and neighboring states (St. Petersburg, World and Family, 990, 1995)

11. R.V. Kamelin, Red Book of the Russian Federation (plants and fungi) (M.: Partnership of Scientific Publications, 855, 2008)

12. Program and methodology of observations of coenopopulations of plant species of the Red Book of the USSR. (M., Gosagroprom of the USSR, 34-36, 1986)

13. V.V. Savelyeva, Nature of the city of Stavropol (Stavropol: Stavropol: Service School, 83$84,2002)$

14. V.G. Tanfilev, Meadow and forbs-sod-grass steppes of the Stavropol upland, Steppes and meadows of the Stavropol Territory: proceedings of the Stavropol Research Institute of Rural Agriculture (Stavropol, SNIISKH, 16-23, 1980)

15. D.S. Dzybov, N.G. Lapenko, Zonal and secondary beard grass steppes of the Stavropol Territory (Stavropol, Kraevaya tipografiya, 224, 2003) 